\title{
Perspective
}

PERSPECTIVE Actualité en histoire de l'art

2| 2007

La Grande-Bretagne/Période moderne

\section{Travaux récents en Allemagne sur l'art dans la France de Louis XIV}

\section{Milovan Stanic}

\section{(2) OpenEdition}

1 Journals

\section{Édition électronique}

URL : http://journals.openedition.org/perspective/3842

DOI : $10.4000 /$ perspective.3842

ISSN : 2269-7721

Éditeur

Institut national d'histoire de l'art

\section{Édition imprimée}

Date de publication : 30 juin 2007

Pagination : 387-392

ISSN : 1777-7852

\section{Référence électronique}

Milovan Stanic, "Travaux récents en Allemagne sur l'art dans la France de Louis XIV », Perspective [En ligne], 2 | 2007, mis en ligne le 31 mars 2018, consulté le 01 octobre 2020. URL : http://

journals.openedition.org/perspective/3842 ; DOI : https://doi.org/10.4000/perspective.3842

Ce document a été généré automatiquement le 1 octobre 2020. 


\title{
Travaux récents en Allemagne sur l'art dans la France de Louis XIV
}

\author{
Milovan Stanic
}

\section{RÉFÉRENCE}

Jutta Held, Französische Kunsttheorie des 17. Jahrhunderts und der absolutistische Staat. Le Brun und die ersten acht Vorlesungen an der königlichen Akademie, Berlin, Reimer, 2001. 421 p., 7 p. coul. ISBN-13 : 978-3496012337; $49 €$.

Pablo Schneider, Phillip Zitzlsperger éd., Bernini in Paris. Das Tagebuch des Paul Fréart de Chantelou über den Aufenthalt Gianlorenzo Berninis am Hof Ludwigs XIV, Berlin, AkademieVerlag, 2006. 500 p., 80 fig. n. et b. ISBN-13 : 978-3050041629; $55 €$.

Thomas Kirchner, Der epische Held. Historienmalerei und Kunstpolitik im Frankreich des 17. Jahrhunderts, Munich, Fink, 2001. 528 p., 117 fig. n. et b, 10 fig. coul. ISBN :

3-7705-3397-6; 94,80€.

Dietrich Erben, Paris und Rom. Die staatlich gelenkten Kunstbeziehungen unter Ludwig XIV, Berlin, Akademie-Verlag, 2004. 409 p., 127 fig. n. et b. ISBN-13 : 978-3050038513 ;

$69,80 €$.

1 À demander aux historiens de l'art allemands spécialistes du Grand Siècle pourquoi ils se préoccupent davantage de l'art français que de l'art en Allemagne, on se verra assurément répondre que le terrain de l'art allemand de cette époque n'offre pas suffisamment de matière d'investigation. Cette appréciation est évidemment exagérée, tous les pays germaniques n'ayant pas été à l'époque aussi dépourvus que cela de créations artistiques de valeur, et elle n'explique en rien pourquoi la majorité des études, et ce depuis longtemps, a été consacrée aux relations entre l'art et le pouvoir, à la politique artistique, aux institutions artistiques et à leurs influences sur le système de représentation du régime monarchique ${ }^{1}$, beaucoup moins souvent aux monographies (si l'on exclut les cas tout à fait exceptionnels de Poussin, de Claude Perrault $^{2}$, de Pierre Puget ou de Girardon ${ }^{3}$ ). Le recours explicite aux ouvrages bien 
connus de Norbert Élias sur la société de cour et ses cérémonials ${ }^{4}$ est caractéristique de telles approches.

Il est vrai que l'Allemagne a été à l'avant-garde de la recherche sur l'iconographie politique, les travaux d'Aby Warburg du début du $\mathrm{xx}^{\mathrm{e}}$ siècle en témoignent. Mais le nom de Warburg, dont l'approche anthropologique n'a plus cours actuellement dans ce domaine, du moins explicitement, n'apparaît pas dans les bibliographies des publications récentes, contrairement à celui de Peter Burke, tout à fait sensible aux recherches anthropologiques. The Fabrication of Louis XIV (1992) ${ }^{5}$, avec le concept de " mise en scène du politique » et le Roi Soleil comme « cas d'école pour le thème de la production des grands hommes ", s'est imposé comme une référence incontournable. Il est tout à fait significatif qu'un ouvrage comme celui de Stephan Germer sur André Félibien ${ }^{6}$, dont il n'existe, loin s'en faut, aucun équivalent en France, ait été critiqué de ce point de vue. La démonstration de Germer (qui se présente en partie comme une contribution à une "théorie des conditions médiatiques du pouvoir absolutiste»), ancrée dans l'analyse structurale, explique comment les formes discursives développées par Félibien pouvaient ramener la production artistique de l'époque au monarque absolu et ainsi leur donner un aspect unifié ; elle expose aussi comment les stratégies d'écriture de Félibien s'émancipent progressivement de leurs attaches thuriféraires pour gagner une autonomie scientifique. Cette démonstration a été sérieusement remise en question, arguant du fait que sous les exigences d'un tel système de représentation surdéterminant, les espaces de liberté auraient été quasi inexistants?

3 La pointe critique surprend moins lorsqu'on sait qu'elle vient de Jutta Held, auteur d'un ouvrage dont le titre est en soi tout un programme: Französische Kunsttheorie des 17. Jahrhunderts und der absolutistische Staat. Le Brun und die ersten acht Vorlesungen an der königlichen Akademie [L'art français du XVII siècle et l'État absolu. Le Brun et les huit premières conférences à l'Académie royale] $]^{8}$. Pour la première fois en Allemagne, les conférences de l'Académie royale résumées par Félibien sont publiées intégralement ${ }^{9}$, accompagnées d'une traduction, commentées et interprétées une par une. Pour J. Held, le projet déterminant de la phase initiale du règne personnel de Louis XIV aurait été la restructuration de la société "dans le sens des tendances absolutistes», la hiérarchisation et l'orientation morale, politique et esthétique de celles-ci vers la monarchie. À aucun moment, le discours esthétique n'aurait abouti à un système théorique autonome, la théorie, à laquelle œuvrait l'Académie royale de peinture et de sculpture, ayant toujours été inextricablement "mêlée à l'histoire» (p.13). Aussi, $\mathrm{J}$. Held définit-elle la finalité de son ouvrage comme la démonstration, à partir des conférences académiques de 1667 et de 1668 (la huitième et dernière de son ensemble), des liens entre la théorie de l'art et la forme de l'État. Le rôle principal dans l'affaire est, comme le signale le sous-titre de l'ouvrage, attribué à Le Brun, « de fait le maître absolu (absolutistisher Herrscher) au sein de l'Académie »; en ce qui concerne le théorème principal du discours esthétique, celui réglant le problème de la composition, selon $\mathrm{J}$. Held, et qui organiserait de manière strictement hiérarchisée les relations entre les parties d'une œuvre et leur subordination au tout, il refléterait parfaitement les volontés du pouvoir absolutiste dans le domaine de l'organisation sociale. Cette question est également manifeste dans les discussions qui suivent les conférences, que ce soit par le débat, pour Held prédominant, entre Le Brun et Philippe de Champaigne ce qui explique l'ajout de la conférence de Champaigne sur Eliezer et Rebecca aux 
conférences éditées par Félibien -, ou dans les concepts-clés exposés à l'Académie tels que bienséance, vraisemblance, ordonnance, où l'apparentement avec les structures sociales et les théories de l'État absolutistes se montreraient avec toute l'évidence souhaitable. Bref, en dépit de maintes observations dans les détails suggérant une vie plus complexe de la Compagnie des peintres et des opinions plus nuancées de ses membres, le cadre conceptuel de Held ne leur laisse aucune liberté d'action entre, d'un côté, le déterminisme téléologique du discours esthétique qui aspirerait, quoique ici encore vainement, à l'autonomie et, de l'autre, le déterminisme politico-économique, assujettissant les artistes aux intérêts de l'État absolu.

4 En général, on peut observer une tendance outre-Rhin à publier des textes importants de la littérature artistique du Grand Siècle français. C'est le cas par exemple avec la récente réédition du Journal de voyage du cavalier Bernin en France, donnée dans une traduction nouvelle et accompagnée d'un nombre important d'articles qui abordent les divers aspects du texte ${ }^{10}$. Julius von Schlosser, malgré ses réserves à l'égard d'une histoire de l'art orientée vers les vies d'artistes, trouvait de l'intérêt au Journal pour ce qu'il contenait de vivant et d'immédiat sur la «vie du grand homme». À partir d'un intérêt élargi aux complexités des relations entre l'art et le pouvoir, aux formes et fonctions de l'art de cour sous la monarchie absolue, le Journal peut enfin être exploité en ce sens comme une source de tout premier ordre ${ }^{11}$. Ainsi sont abordés dans neuf articles, sur 160 pages de la nouvelle édition, les cours de Rome et de Paris (Volker Reinhardt), les turbulences diplomatiques autour de l'affaire des gardes corses en relation avec le voyage (Arne Karsten), la conversation sur l'art saisie entre les nouvelles formes de civilité courtoise et la raison d'État (Wolfgang Brassat), la collection d'art de Chantelou et la postérité de Poussin (Matthias Bruhn), le Bernin et ses commanditaires français (Dietrich Erben), Chantelou et l'écriture sur les œuvres d'art en France aux XVII et XVII ${ }^{e}$ siècles (Thomas Kirchner), les controverses autour du buste de Louis XIV (Philipp Zitzlsperger), les positions rivales de Le Brun et du Bernin au sujet de l'imagination artistique entre ce qui est enseignable et ce qui ne l'est pas (Pablo Schneider).

5 Deux auteurs parmi ceux qui ont contribué à cette nouvelle et très stimulante approche du Journal, Thomas Kirchner et Dietrich Erben, ont publié d'importants ouvrages sur l'art et la politique artistique en France au XVII ${ }^{\mathrm{e}}$ siècle. Dans les deux cas il s'agit de contributions riches, où abondent les études souvent très détaillées de la production artistique et de son contexte, ainsi que d'innovantes constructions conceptuelles.

6 Thomas Kirchner $^{12}$ ouvre dans son livre un vaste champ d'interrogations sur la peinture d'histoire en France au XvII e siècle, traçant l'évolution du genre avec ses fondements théoriques et stylistiques. Chronologiquement situé entre le règne d'Henri IV et les désagrégations politique et culturelle des premières années du XVII ${ }^{\mathrm{e}}$ siècle, son étude, avec l'accent mis sur les cycles décoratifs relatifs à l'histoire dynastique et nationale, met en relation la galerie des portraits d'Henri IV au Louvre et la galerie des Glaces de Versailles, passant par la riche histoire des échanges, complexes, entre l'art italien et l'art français, par l'évolution de la " peinture épique " pensée et pratiquée par les peintres de l'Académie royale, par le cycle des batailles d'Alexandre de Le Brun, par les débats autour d'une représentation adéquate, historiographique ou allégorique, de l'histoire contemporaine, etc. Kirchner prend le titre de l'ouvrage de Warnke, L'artiste de cour : aux origines de l'artiste moderne, au pied de la lettre et le précise, à sa manière, à travers plus de 450 pages, développant sa conception particulière du sens du terme 
«politique artistique » à l'époque de Louis XIV : loin de s'opposer, comme le veut une vision romantique de la relation de l'art et de la politique, les deux domaines, art et politique, seraient, d'après Kirchner, tellement liés l'un à l'autre dans cette période de grandes ambitions étatiques et monarchiques, où naît également en France une théorie de l'art et des institutions artistiques dignes de ce nom, qu'il ne conviendrait pas de les penser en termes d'opposition, mais de complicité. La politique artistique - on devrait parler plutôt de la politique d'art - signifierait à l'époque certes une mainmise du pouvoir sur la production artistique, mais favorisant, dans son propre intérêt, les qualités proprement artistiques de l'art et de sa théorie. "La politique », dit Kirchner, « n'avait pas seulement besoin de l'art en général pour son autoreprésentation, elle demandait un art disposant d'un caractère décidément artistique [...]. Et l'art avait besoin de cette impulsion venant du côté de la politique, pour entamer un discours, dont le centre n'était plus occupé par l'œuvre politiquement déterminée, mais par le tableau de chevalet à tendance autonome» (p.11). Symbiose, donc, plutôt qu'antagonisme, entre la politique et l'art, celle-là profitant du déplacement de l'intérêt du contenu vers la forme artistique, celui-ci profitant des besoins de la représentation politique pour préciser la réflexion sur les genres, notamment sur le support du tableau de chevalet et sur les conceptions clés de sa théorie, les notions de vrai et de vraisemblable. Le haut lieu de la convergence des intérêts aurait été l'Académie royale de peinture et de sculpture où, pour un temps, les affinités entre l'art et la politique auraient trouvé un langage commun. Après, les dissonances auraient à nouveau repris le dessus, la crisis ne tardant pas à survenir : le moment où les programmes décoratifs, à Versailles (à partir de 1674), ne seront plus définis par la volonté politique à partir des traditions de la narration épique, mais de l'histoire factuelle.

7 L'ouvrage de Dietrich Erben, tout aussi riche et bien documenté que celui de Kirchner, issu d'une thèse d'habilitation, est concentré sur le rôle de l'Italie, notamment de Rome, dans la politique artistique de la France de Louis XIV. Cela ne va évidemment pas sans d'amples développements, très détaillés et érudits, consacrés aux campagnes des Français en Italie à la Renaissance, aux acquisitions et autres investissements de François I Ir, Henri IV, Louis XIII, etc. Cependant, selon Erben, tous ces efforts, actions, finesses diplomatiques, mises en scène ostentatrices et fastes pompeux employés pour doter la France d'un appareil représentatif digne de son rang dans le concert des nations européennes ne parvinrent pas, avant le règne de Louis XIV, à en faire une politique culturelle réelle et cohérente. Cette dimension nouvelle serait due à ce qu'Erben considère comme le primo mobile de la politique royale en général : la politique expansionniste visant l'établissement de la "monarchie universelle». Ce serait cette dernière instance, une prétention, surambitionnée, à la prééminence en Europe, qui aurait déterminé et unifié, en quelque sorte, la production de l'art commandé pour les besoins de représentation de la couronne.

8 L'obstacle sur le chemin de la monarchie universelle est Rome, siège ancestral du pouvoir impérial dans l'Antiquité et siège du pouvoir spirituel actuel de la papauté. Les stratégies de la politique artistique du Roi Soleil suivraient la finalité de l'expropriation de la Ville Éternelle de ces prérogatives, stratégies dont Erben suit les étapes au fil des chapitres : retrouver le niveau d'échanges artistiques atteint naguère sous François I ${ }^{\mathrm{er}}$; débaucher le premier artiste d'Alexandre VII, Bernin, pour le mettre au service du monarque français; s'approprier, par acquisition ou par copie, le meilleur de la production artistique romaine, antique et moderne; s'assurer de la place représentative à Rome supérieure à celle des autres monarchies, surtout de celle des 
Habsbourg ; intégrer dans la production artistique française les modèles italiens, en les surpassant.

Un bon exemple de la méthode d'Erben réside dans son interprétation de la façade Est du Louvre, décidée à la place du projet berninien : contrairement à la vision du palais d'Apollon, proposée naguère par Michael Petzet dans son ouvrage sur Claude Perrault ${ }^{13}$, Erben y voit «l'émancipation de l'architecture romaine contemporaine, représentée par Bernin » et « l'orientation explicite à l'héritage de la Rome antique et à celui de la Renaissance française » dans le contexte de la guerre de Dévolution (1667-1668). Car avec la guerre, la fonction du Louvre a changé : il ne s'agissait plus d'une résidence pour le roi mais, "comme le montre la façade", c'est-à-dire le motif de l'arc de triomphe du pavillon central, de "l'appropriation synthétisante [de l'architecture antique] au profit d'une représentation universaliste » (p. 108).

L'aspiration, rendue visible, à une monarchie universelle: elle pourrait expliquer le choix triomphal, à supposer que cette aspiration ait réellement inspiré les actions de Louis XIV à l'époque; elle expliquerait plus difficilement, pourrait-on objecter, le désintérêt du roi pour son palais dans la capitale et l'adoption définitive, comme résidence principale, du « château de plaisir » qu'était Versailles selon l'expression de Colbert, au moment même des débats. Un désaccord aussi profond au sommet ? Colbert aurait-il eu une conscience plus aiguë que le roi lui-même de la monarchie universelle et de ses exigences comme programme pour la représentation?

Plus important encore que la façade Est du Louvre pour la démonstration d'Erben, le dôme des Invalides, dont l'interprétation fait l'objet du chapitre final de son ouvrage. Par sa destination de sépulcre du roi, une ancienne thèse reprise par Erben, par ses aspects formels rivalisant avec ceux de Saint-Pierre de Rome, le dôme représenterait la « concurrence au pouvoir universel du pape » avec les moyens de l'art. Le «monument des revendications universalistes » (p. 342), pourrait-on encore objecter, était pourtant laissé par le roi entièrement à la supervision de Louvois qui, dès son vivant, a exprimé le désir de s'y faire inhumer, et rien ne soutient l'hypothèse du dôme comme le futur lieu sépulcral de Louis XIV, hypothèse réfutée, une fois pour toutes espérons-le, par les récentes publications de Thierry Sarmant ${ }^{14}$. La relation à Rome fut certainement un élément capital dans l'ensemble de la politique de prestige de Louis XIV. Mais reflète-telle pour autant l'aspiration à une monarchie universelle ? Il est permis d'en douter.

Dans le cas de Thomas Kirchner, la distinction stricte entre "dimension politique » d'un côté, et «cadre artistique » de l'autre, paraît trop artificielle, en tout cas trop conceptuellement abstraite, pour guider les lectures de certaines œuvres de manière convaincante ; tout comme la distinction du " public » et du "privé » dans le cadre de l'espace versaillais (public serait l'escalier des Ambassadeurs, privés les Grands appartements du roi). Le concept moderne d'art ne semble pas plus applicable aux œuvres $\mathrm{du} \mathrm{xVII}^{\mathrm{e}}$ siècle que celui du privé aux espaces de la résidence royale à Versailles $^{15}$.

L'aporie paraît manifeste, au plus tard dans la conclusion de Kirchner, où un tableau de Watteau, Les comédiens italiens (Washington, National Gallery of Art), figure pour démontrer la fin de l'association transitoire entre la " politique artistique programmée au nom du roi » et le « cadre artistique » : la composition montre Pierrot de plain-pied, encadré de personnages dont l'un le montre théâtralement au spectateur. Kirchner y décèle un hiatus, entre "le groupe de personnages noblement habillés " et Pierrot, occupant la position centrale qui, «dans une composition classique, marque celle du 
héros » (p. 462). Mais, s'agissant des comédiens italiens - on reconnaît immédiatement les personnages fixes d'Arlequin, de Colombine, du Docteur, etc. -, le spectateur n'attend pas un "héros classique " à la place de Pierrot, son "attente" n'est pas « déçue » (p. 463) : on n'attend pas plus trouver un Énée classique dans le Virgile travesti de Scarron.

Les études dont il est question ici, surtout celles de Germer, de Kirchner et d'Erben, ne laissent pas pour autant d'être précieuses à plus d'un titre. Les auteurs ont assimilé les recherches, françaises et autres, pour leur plus grande part et offrent de leur côté de nouvelles pistes fondées sur un grand nombre de faits historiques et artistiques souvent négligés ou insuffisamment étudiés. À tout le moins, on ne saurait ni leur reprocher de négliger l'histoire factuelle, ni non plus d'être de simples collecteurs de faits. Le problème se situe ailleurs, comme nous tentions de le suggérer en soulevant quelques points critiques. Il est, nous semble-t-il, hérité de la longue tradition allemande de la philosophie de l'histoire: c'est le problème de la conceptualisation monocausale, encombrante déjà comme telle, mais ambiguë lorsqu'il s'agit de passer de la constitution d'une structure stable à l'action, ou au changement; par exemple, du système colbertiste à sa désagrégation sous Louvois, puis à sa reconstitution plus tard. Cela amène par ailleurs le danger d'un argument circulaire, d'attribuer à l'«absolutisme » ou à la «monarchie universelle » tout ce qui provoque les formes et les événements étudiés. Dans quelle mesure les hommes peuvent-ils manipuler une structure politique stable telle que l'"absolutisme $»^{16}$ ? Ou des concepts organisateurs tels que "forme artistique", qui s'applique tantôt à des idées, tantôt à des formes concrètes d'œuvres? Ces questions ne sont pas nouvelles, certes, mais elles demeurent actuelles pour un travail historiographique qui se réclame souvent du new historicism, mais sans se débarrasser des tendances déterministes, comme celui-ci le recommande.

Il ne saurait être question ici de recommander à la recherche allemande en histoire de l'art un retour au "comment ce fut en effet - wie es denn gewesen ist» de Ranke, de réviser en quelque sorte son critique Burckhardt; en revanche, un peu moins de zèle hypothético-déductif, un peu plus de pluralisme explicatif dans la constitution de l'inventaire et du savoir historique seraient bienvenus. Quant à la recherche française, elle ferait bien de prendre davantage en compte celle d'outre-Rhin, et pas seulement ses éléments spéculatifs, pour stimuler ses propres questionnements.

\section{NOTES}

1. L'optique politique guide souvent des travaux consacrés aux œuvres singulières ou aux formes typologiques, telles l'étude de Kirsten Ahrens, Hyacinthe Rigauds Staatsporträt Ludwigs XIV. Typologische und ikonologische Untersuchung zur politischen Aussage des Bildnisses von 1701 (Worms, 1998), ou celle d'Andreas Köstler, Place Royale : Metamorphosen einer kritischen Form des Absolutismus (Munich, 2003). C'est encore vrai pour la volumineuse étude de Marion Bornscheuer consacrée en 
2005 à la «théorie de la peinture » de Sébastien Bourdon, où l'orientation politique, culturelle et religieuse joue un rôle déterminant (voir notre recension dans la Revue de l'art, 151, 2006-1, p. 81 suiv.). Cette orientation surprend moins lorsqu'il s'agit des analyses de fêtes de cour et de grandes cérémonies, à commencer par l'excellent ouvrage de Karl Möseneder, Zeremoniell und monumentale Poesie : Die 'Entrée solennelle' Ludwigs XIV. 1660 in Paris, qui remonte à 1983, ou par des contributions dans Geselligkeit und Gesellschaft im Barockzeitalter, vol. 2, «Wolfenbütteler Arbeiten zur Barockforschung", 28.2, Wiesbaden, 1997 (des recherches novatrices sont consacrées actuellement aux fêtes versaillaises, à l'Université de Francfort). Cette question est le sujet même de l'ouvrage de synthèse d'Alexandra Bettag, Die Kunstpolitik Jean Baptiste Colberts. Unter besonderer Berücksichtigung der Académie royale de peinture et de sculpture (Weimar, 1998). Sur l'Académie royale d'architecture, signalons l'ouvrage remarquable de Wolfgang Schöller, Die 'Académie Royale d'Architecture' 1671-1793 : Anatomie einer Institution, Cologne, 1993.

2. Michael Petzet, Claude Perrault und die Architektur des Sonnenkönigs : Der Louvre König Ludwigs XIV. und das Werk Claude Perraults, Munich/Berlin, 2000.

3. Sur Puget, Klaus Herding prépare une nouvelle édition de sa monographie de référence. Sur Girardon, voir Adam Klidis, François Girardon : Bildhauer in königlichen Diensten 1663-1700, Weimar, 2001.

4. Les travaux d'Elias continuent à susciter de nouveaux débats et des recherches, voir par exemple Claudia Opitz, éd., Höfische Gesellschaft und Zivilisationsprozess : Norbert Elias' Werk in kulturwissenschaftlicher Perspektive, Cologne, 2005.

5. Trad. fr. : Peter Burke, Louis XIV, Les stratégies de la gloire, Paris, 1995. On devrait joindre au nom de Burke ceux de Kantorowicz et d'Apostolidès.

6. Stefan Germer, Kunst, Macht, Diskurs. Die intellektuelle Karriere des André Félibien im Frankreich von Louis XIV, Munich, 1997.

7. Jutta Held, dans Kunstchronik, 52, 1999, p. 259-263.

8. Des études sur l'Académie royale ont cours actuellement. Voir, à part les ouvrages déjà mentionnés : Wilhelm Schlink, Ein Bild ist kein Tatsachenbericht. Le Bruns Akademierede von 1667 über Poussins Mannawunder, Freibourg-im-Brisgau, 1996 ; Cathrin Klingsöhr-Leroy, Das Künstlerbildnis des Grand Siècle in Malerei und Graphik. Vom « Noble Peintre » zum « Pictor Doctus », Munich, 2002.

9. J. Held a ajouté aux sept conférences publiées par Félibien la huitième, celle de Philippe de Champaigne sur Eliezer et Rebecca de Poussin, transmise dans un résumé par Guillet de SaintGeorges. Les sept premières conférences sont données d'après l'édition Trévoux de 1725, et non d'après les éditions du XvII ${ }^{\mathrm{e}}$ siècle.

10. La nouvelle traduction est fondée sur celle de Hans Rose (1919), littérairement très séduisante mais assez éloignée de l'original français, et suit l'édition critique française du Journal (Milovan Stanic éd., Paris, 2001).

11. Quelques prémices de cette lecture se trouvent déjà chez Martin Warnke, L'artiste et la cour: aux origines de l'artiste moderne, Paris, 1989 (Hofkünstler. Zur Vorgeschichte des modernen Künstlers, Cologne, 1985).

12. Certains aspects de l'ouvrage de Kirchner furent déjà abordés par lui dans sa publication L'expression des passions. Ausdruck als Darstellungsproblem in der französischen Kunst und Kunsttheorie des 17. und 18. Jahrhunderts, Mayence, 1991.

13. Voir Petzet, 2000, cité n. 2.

14. L'ouvrage d'Erben a été recensé dans le même sens, avec davantage de détails, par H. Ziegler, Rezension von: Dietrich Erben: Paris und Rom. Die staatlich gelenkten Kunstbeziehungen unter Ludwig XIV, Berlin, Akademie Verlag 2004, dans sehepunkte 5 (2005), Nr. 4 [15.04.2005] (http:// www.sehepunkte.historicum.net/2005/04/5473.html).

15. Une recension a ainsi recommandé de lire l'ouvrage dans le sens d'une reconstruction des conceptions hétéronomes, concurrentes, de l'image à l'âge classique, plutôt que dans le sens de l'« histoire des débuts d'un art luttant pour son autonomie» (Kirchner, p. 15 et 464). Voir J. Rees, 
Rezension de : Thomas Kirchner, Der epische Held. Historienmalerei und Kunstpolitik im Frankreich des 17. Jahrhunderts, Munich, Fink, 2001, dans sehepunkte 2 (2002), Nr. 6 [15.06.2002] (http:// www.sehepunkte.historicum.net/2002/06/3770533976.html)

16. Les études ne prennent en général pas suffisamment en considération les récentes recherches historiques sur l'absolutisme et conçoivent celui-ci comme l'exercice d'un despotisme illimité.

\section{INDEX}

Index géographique : Allemagne, France

Keywords : german art, art history, historiography, political iconography, academy, monarchy Mots-clés : art allemand, histoire de l'art, historiographie, iconographie politique, académie, monarchie

Index chronologique : 1600

\section{AUTEURS}

\section{MILOVAN STANIC}

Université de Paris IV-Sorbonne 